\title{
ИССЛЕДОВАНИЕ АВТОМАТИЗИРОВАННОЙ СИСТЕМЫ НАТЯЖЕНИЯ РЕМНЕЙ ПРИВОДА СТАНКА-КАЧАЛКИ
}

\author{
Уразаков Камил Рахматуллович', \\ Urazakk@mail.ru
}

Тимашев Эдуард Олегович', timashev@mail.ru

\author{
Шайжанов Нурсултан Серикболатович', \\ shaizhanov007@yandex.ru \\ Тугунов Павел Михайлович', \\ Paveltugunov@gmail.com \\ 1 Уфимский государственный нефтяной технический университет, \\ Россия, 450062, г. Уфа, ул. Космонавтов, 1.
}

\begin{abstract}
Актуальность. Работоспособность балансирных станков-качалок - наиболее распространенного типа привода скважинных штанговых насосов - в значительной степени определяется надежностью работы клиноременных передач. Отсутствие механизма, обеспечивающего оптимальное натяжение приводных ремней, обуславливает потери энергии в клиноременной передаче за счет проскальзывания ремней и снижение эксплуатационных характеристик насосных установок. В настоящее время контроль и регулировка натяжения приводных ремней станка-качалки производятся вручную. Вышесказанное обуславливает актуальность разработки механизмов автоматического натяжения ремней.

Объект: автоматический натяжитель приводных ремней станка-качалки, использующий принцип регулирования межосевого расстояния шкивов путем изменения угла наклона платформы, на которой установлен электродвигатель. Регулирование угла наклона достигается совместной работой пружин сжатия разной жесткости. Пружина большей жесткости за счет силы упругой деформации сжатия обеспечивает натяжение ремней, пружина меньшей жесткости предназначена для компенсации колебаний в системе.

Цель: исследование автоматизированной системы натяжения ремней привода станка-качалки, разработка методики расчета автоматического натяжителя ремней, включающая определение оптимальной величины угла наклона платформы к горизонтали, положения электродвигателя на платформе, упругих характеристик пружин.

Методы: применение принципов аналитической механики, дифференциального исчисления для расчета оптимальных величин параметров автоматического натяжителя.

Результаты. Разработана методика расчета автоматического натяжителя приводных ремней станка-качалки, позволяющая поддерживать оптимальную величину натяжения ремней в процессе работы штанговой насосной установки. Рассчитаны оптимальные величины начального угла наклона платформы, положения электродвигателя на платформе, пределы изменения угла наклона платформы в процессе растяжения ремней. Показано, что с точки зрения минимизации нагруженности пружины наиболее оптимальными являются: минимальное значение угла наклона платформы и максимальное расстояние вдоль нее до электродвигателя. Произведен расчет пружин сжатия, входящих в компоновку автоматического натяжителя, определены оптимальные значения коэффициентов жесткости пружин.
\end{abstract}

\section{Ключевые слова:}

Станок-качалка, приводной ремень, натяжение ремней, автоматический натяжитель, пружина сжатия, коэффициент упругости пружины.

\section{Введение}

В последнее время в связи с сокращением объемов добычи нефти и ростом числа мало- и низкодебитных скважин, обуславливающим увеличение доли добывающего фонда, эксплуатируемого установками скважинных штанговых насосов (УСШН), значительное внимание уделяется разработке методов и технологий, направленных на повышение эффективности насосной эксплуатации. Наиболее актуальными задачами рентабельной разработки механизированного фонда являются: обеспечение достижения технологического потенциала скважин за счет обоснованного выбора оптимального режима эксплуатации и увеличение периода безотказной работы насосного оборудования [1-6].
Эффективность работы штанговых насосных установок в значительной степени определяется надежностью наземного привода. Одним из путей обеспечения стабильной работы балансирных станков-качалок (CK) - наиболее распространенного типа привода скважинных штанговых насосов - является повышение надежности клиноременных передач. В процессе эксплуатации станков-качалок натяжение приводных ремней изменяется вследствие воздействия различных факторов. В частности, изменение температуры окружающей среды обуславливает изменение длины ремней и величины натяжения за счет изменения физических свойств материала ремней. Существенное влияние на состояние ремней оказывает временной фактор, который приводит к растяжению 
и ослаблению натяжения ремней с течением времени [7-12].

Отсутствие в кинематике станка-качалки элемента, компенсирующего недостаточное или избыточное натяжение ремней, способствует снижению дебита скважины, необоснованным потерям энергии в клиноременной передаче и снижению КПД насосных установок. В настоящее время регулировка натяжения ремней производится вручную и требует полной остановки работы насосной установки. Вышеперечисленное обуславливает актуальность разработки механизмов, позволяющих поддерживать натяжение ремней СК на оптимальном уровне на протяжении всего периода работы УСШН.

Для предотвращения ослабления натяжения предложен механизм автоматического натяжителя приводных ремней СК, использующий принцип регулирования межосевого расстояния шкивов путем изменения угла наклона платформы, на которой установлен электродвигатель. Компоновка натяжителя включает в себя наклонную платформу из швеллеров с установленным на ней асинхронным электродвигателем (рис. 1). С одной стороны платформа закреплена на упруго-деформируемой опоре, которая может растягиваться или сжиматься за счет применения двух пружин, расположенных с противоположных сторон относительно плоскости платформы. С другой стороны платформа шарнирно закреплена на раме.

Регулирование угла наклона достигается совместной работой пружин сжатия разной жесткости, у каждой из которых один конец закреплен, а второй взаимодействует с платформой. Натяжение ремней обеспечивается силой упругой деформации верхней пружины большей жесткости (индекс 1) совместно с весом электродвигателя и платформы. Результирующий момент данных сил относительно неподвижнной оси вращения платформы направлен противоположно моменту, обусловленному силой натяжения ремней. В случае возможного ослабления натяжения ремней происходит их дополнительное растяжение, что позволяет обеспечить постоянную величину натяжения.

Нижняя пружина меньшей жесткости (индекс 2) имеет противоположное направление усилия по отношению к пружине большей жесткости и служит для повышения устойчивости и надежности работы автоматического натяжителя. Известно, что вращение вала электродвигателя вызывает возникновение центробежных сил за счет неуравновешенных масс на шкиве. Уменьшение угла наклона платформы в результате действия сил инерции обуславливает дополнительное сжатие нижней пружины и возникновение возвращающей силы, стремящейся вернуть платформу в первоначальное положение.

Механизм автоматического натяжения, в котором передней опорой стола электродвигателя служит шток с установленной на нем парой пружин для обеспечения постоянного натяжения ремней клиноременной передачи, причем ресурс пружин расходуется на натяжение ремней и преодоление веса платформы и электродвигателя, разработан в [1]. За счет использования собственной массы электродвигателя и платформы для поддержания натяжения ремней предложенный вариант требует меньшую нагруженность пружин и является более эффективным, поскольку позволяет значительно меньше использовать ресурс пружин, а значит способствует увеличению эксплуатационной надежности механизма натяжения.

\section{Расчет оптимальных величин угла наклона платформы и положения электродвигателя на платформе}

Методика расчета автоматического натяжителя ремней СК включает расчет оптимальных значений угла наклона платформы к горизонтали $\alpha$, положения электродвигателя на платформе (в качестве независимой переменной примем расстояние от неподвижной оси вращения платформы до линии, перепендикулярной плоскости платформы и проходящей через центр масс электродвигателя $L_{\text {эд }}$, которое в дальнейшнем обозначается термином «расстояние вдоль платформы до электродвигателя») и упругих характеристик пружин (коэффициентов упругости, силы упругой деформации), удовлетворяющих следующим основным критериям:

1) Постоянство величины натяжения ремней СК в процессе изменения межосевого расстояния шкивов и угла наклона платформы

2) Обеспечение устойчивой работы автоматического натяжителя при минимальной нагруженности его узлов.

Уравнение моментов относительно точки 0 (рис. 1):

$$
\begin{gathered}
\sum M=F_{\text {пр }} L_{\text {пр }}-S_{\Sigma} h_{2} \sin \beta-S_{\Sigma} h_{1} \cos \beta+ \\
+m_{\text {әд }} g h_{2}+m_{\text {ст }} g h_{3}=0,
\end{gathered}
$$

где $F_{\text {пр }}$ - результирующая сила упругости системы пружин; $m_{\text {эл }}$ - масса асинхронного электродвигателя; $m_{\text {сг }}$ - масса платформы; $g$ - ускорение свободного падения; $S_{\Sigma}$ - окружная сила, создаваемая силой натяжения ремней; $L_{\text {пр }}$ - плечо момента результирующей силы упругости пружин; $\beta$ - угол между горизонталью и линией, проходящей через центры осей вращения шкивов редуктора и электродвигателя; $h_{1}$ - плечо момента горизонтальной составляющей силы натяжения ремней; $h_{2}-$ плечо момента силы тяжести электродвигателя; $h_{3}-$ плечо момента силы тяжести платформы. Результирующая сила упругости системы пружин - векторная сумма сил упругой деформации $\sum_{i=1}^{2} \vec{F}_{\text {пр }}^{i}$, создаваемых каждой из пружин [13].

Для нахождения наиболее оптимальных значений варьируемых параметров выразим переменные в (1) через независимые переменные $\alpha$ и $L_{\text {эд }}$. Учтем следующие геометрические связи между исходными и независимыми переменными: 


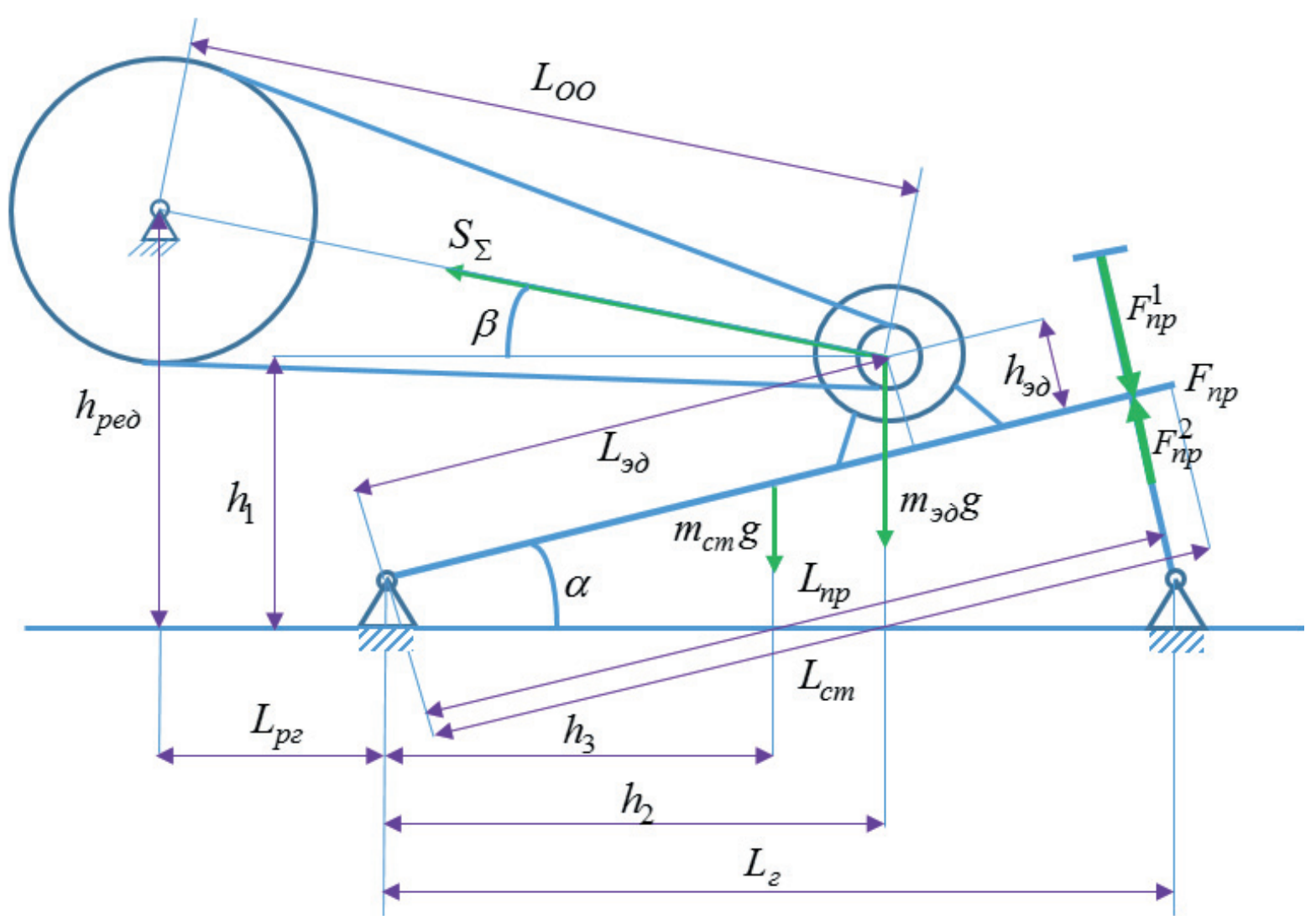

Рис. 1. Схема сил и моментов для автоматического натяжителя ремней

Fig. 1. Scheme of forces and moments of forces for the automatic belt tensioner

$$
\begin{gathered}
h_{1}=L_{\text {әд }} \sin \alpha+h_{\text {эд }} \cos \alpha ; \\
h_{2}=L_{\text {эд }} \cos \alpha-h_{\text {эд }} \sin \alpha ; \\
h_{3}=\frac{L_{\text {ст }}}{2} \cos \alpha ; \\
L_{\text {пр }}=L_{\mathrm{r}} \cos \alpha ; \\
\sin \beta=\frac{h_{\text {ред }}-\left(L_{\text {эд }} \sin \alpha+h_{\text {эд }} \cos \alpha\right)}{L_{\text {оо }}},
\end{gathered}
$$

где $h_{\text {эд }}$ кратчайшее расстояние от платформы до оси вращения шкива электродвигателя («высота» электродвигателя); $L_{\mathrm{r}}$ - расстояние по горизонтали между осями вращения платформы и упруго-деформируемой опоры; $L_{\text {ст }}-$ длина платформы; $h_{\text {ред }}-$ высота оси вращения шкива редуктора; $L_{0}-$ pacстояние между осями вращения шкивов редуктора и электродвигателя. Подставляя выражения (2)-(6) в (1), получим:

$$
\begin{gathered}
F_{\text {пр }} L_{\text {г }} \cos \alpha-S_{\Sigma}\left(L_{\text {эд }} \cos \alpha-h_{\text {эд }} \sin \alpha\right) \sin \beta- \\
-S_{\Sigma}\left(L_{\text {эд }} \sin \alpha+h_{\text {эд }} \cos \alpha\right) \cos \beta+ \\
+m_{\text {эд }} g\left(L_{\text {эд }} \cos \alpha-h_{\text {эд }} \sin \alpha\right)+\frac{m_{\text {ст }} g L_{\text {ст }} \cos \alpha}{2}=0
\end{gathered}
$$

или в более общем виде:

$$
F\left(S_{\Sigma}, L_{\text {ст }}, L_{\Gamma}, m_{\text {эд }}, m_{\text {ст }}, h_{\text {эд }}, h_{\text {ред }}, L_{\text {оо }}, \alpha, L_{\text {эд }}, F_{\text {пр }}\right)=0 .
$$

Уравнения (7) и (8) связывают между собой основные переменные системы: 1) заданные величины, такие как окружная сила, создаваемая силой натяжения ремней, массы электродвигателя и платформы, длина платформы, расстояния между неподвижными осями вращения платформы и упруго-деформируемой опоры и между осями вращения шкивов редуктора и электродвигателя; 2) варьируемые величины, такие как угол наклона платформы к горизонтали, положение электродвигателя на платформе, результирующая сила упругости системы пружин, действующая со стороны пружин на платформу и обеспечивающая необходимое натяжения ремней СК. На рис. 2 показано, как изменяется результирующая сила упругости системы пружин при различных значениях переменных $\alpha$ и $L_{\text {эн. }}$.

Как видно из графика, в зависимости от угла наклона платформы перемещение электродвигателя вдоль платформы приводит к различному изменению результирующей силы упругости системы пружин, необходимой для поддержания постояннного натяжения ремней СК. При малых углах наклона платформы момент силы тяжести, связанный с собственной массой ЭД и платформы, преобладает над моментом силы, действующей на электродвигатель со стороны ремней, поэтому увеличение расстояния вдоль платформы до электродвигателя приводит к возрастанию суммарного момента 


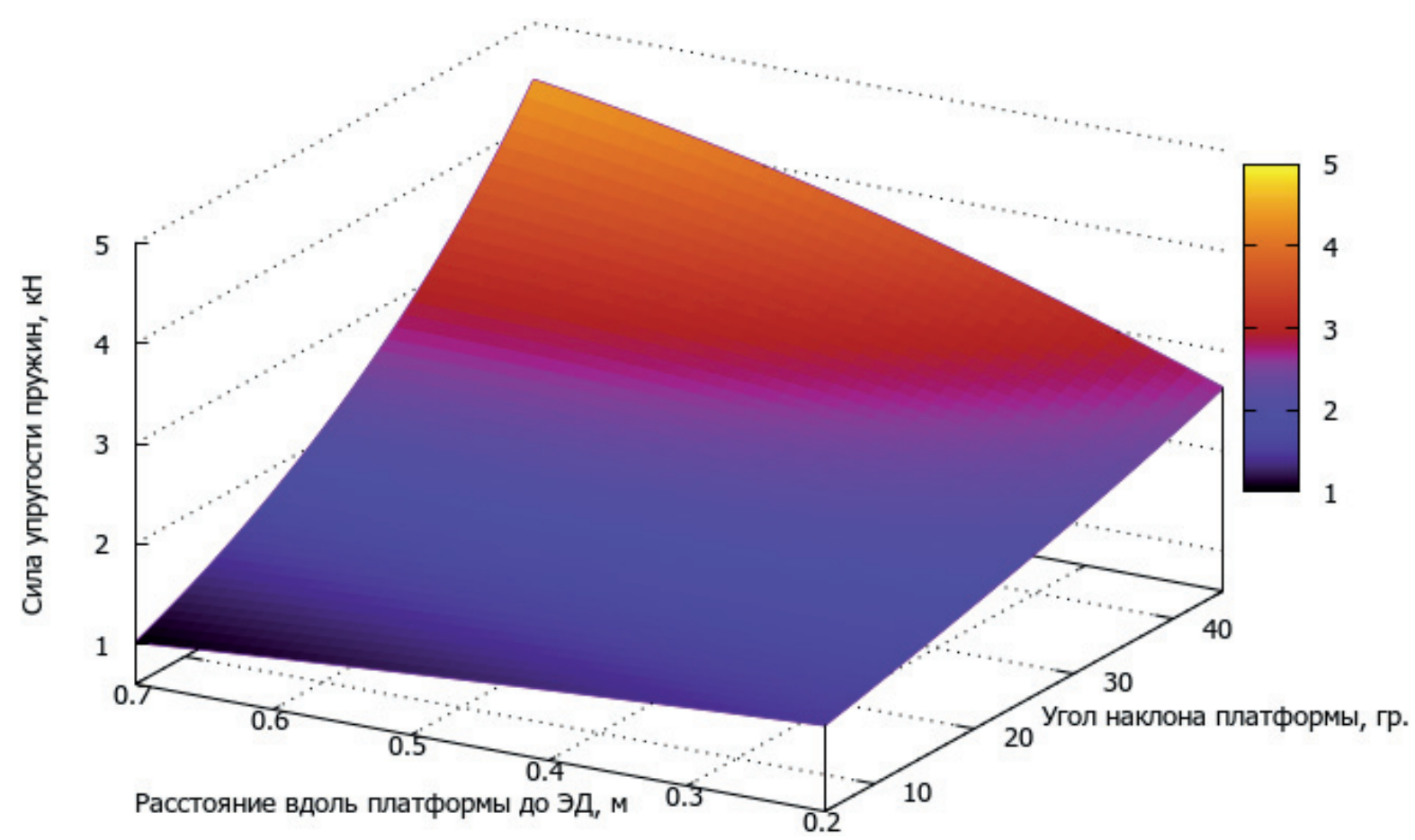

Рис. 2. Зависимость необходимой результирующей силь упругости пружины от начального значения варьируемых параметров (угла наклона платфорлы и положения электродвигателя на платформе)

Fig. 2. Dependence of the necessary resultant spring elasticity on the initial value of the variable parameters (the angle of inclination of the platform and the position of the motor on the platform)

этих сил. Напротив, при больших значениях угла наклона платформы момент сил тяжести оказывается недостаточным для компенсации силы, действующей на электродвигатель со стороны ремней, и с увеличением расстояния ЭД вдоль платформы необходимая результирующая сила упругости системы пружин увеличивается (рис. 3). Для угла наклона платформы $20^{\circ}$ изменения моментов сил при увеличении расстояния от оси вращения платформы до ЭД компенсируют друг друга, и результирующая сила упругости системы пружин практически не зависит от положения ЭД на платформе.

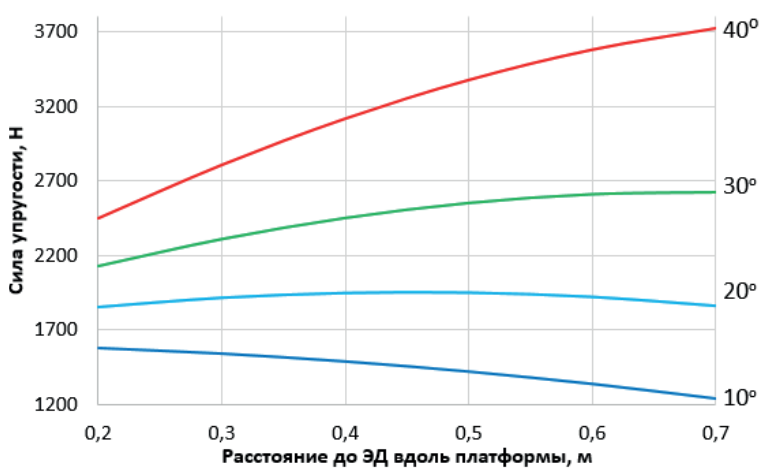

Рис. 3. Зависимость результирующей силы упругости системь пружин от положения электродвигателя на платформе при различных значениях угла наклона платформы

Fig. 3. Dependence of the resulting elastic force of the spring system on the position of the electric motor on the platform for different values of the platform inclination angle
Если задаться некоторым выбранным значением расположения электродвигателя на платформе, то изменение результирующей силы упругости системы пружин будет полностью определяться изменением угла наклона платформы (рис. 4).

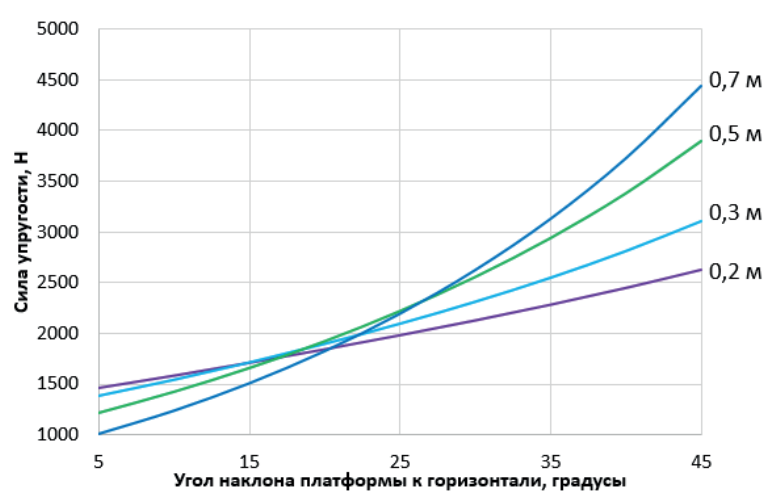

Pис. 4. Зависилость результирующей силь упругости системы пружин от угла наклона платформы при различных положениях электродвигателя на платфорле

Fig. 4. Dependence of the resultant elasticity of the spring system on the platform inclination angle at different positions of the motor on the platform

Из графиков на рисунке видно, что чем дальше расположен электродвигатель на платформе, тем сильнее изменяется с углом результирующая сила упругости пружин, необходимая для поддержания постоянной величины натяжения ремней CK. В области небольших значений угла наклона плат- 
формы к горизонтали (меньше $20^{\circ}$ ) более рациональным является увеличение расстояния вдоль платформы до ЭД, а для больших углов наклона (более $20^{\circ}$ ) это расстояние следует уменьшать. Таким образом, с точки зрения минимизации нагруженности пружины наиболее оптимальными являются: минимальное значения угла наклона платформы и максимальное расстояние вдоль нее до электродвигателя.

\section{Определение области варьирования угла наклона платформы}

В процессе работы привода СК происходит удлинение ремней, и начальный угол наклона платформы изменяется. Область, в которой варьируется значение угла наклона платформы, определяется исходя из величины изменения длины ремней в процессе их растяжения. В системе координат, связанной с неподвижной осью вращения шкива редуктора, координаты оси вращения шкива электродвигателя определяются как

$$
\left\{\begin{array}{l}
x(\alpha)=L_{\text {рг }}+L_{\text {эд }} \cos \alpha-h_{\text {эд }} \sin \alpha ; \\
y(\alpha)=h_{\text {ред }}-L_{\text {эд }} \sin \alpha-h_{\text {эд }} \cos \alpha,
\end{array}\right.
$$

где $L_{\text {рг }}$ - расстояние по горизонтали от оси вращения платформы до линии, проходящей через ось вращения шкива редуктора перпендикулярно плоскости опоры.

Если принять начальное значение угла наклона платформы, при котором ремень не растянут, за $\alpha_{0}$ и конечное значение угла наклона, при котором ремень приобрел максимальное допускаемое удлинение, как $\alpha_{1}$, то разница расстояний между осями вращения шкивов электродвигателя в начальном и конечном положении рассчитывается с учетом (9) согласно формуле

$$
\Delta L_{\mathrm{oo}}=\sqrt{x^{2}\left(\alpha_{1}\right)+y^{2}\left(\alpha_{1}\right)}-\sqrt{x^{2}\left(\alpha_{0}\right)+y^{2}\left(\alpha_{0}\right)} .
$$

$\mathrm{C}$ другой стороны, изменение положения шкива электродвигателя относительно шкива редуктора, связанное с удлинением приводного ремня, можно выразить, зная диаметры шкивов, длину ремня в нерастянутом состоянии и максимальное допускаемое удлинение ремня:

$$
\begin{aligned}
& \Delta L_{\text {оо }}=\sqrt{0,25\left(\begin{array}{l}
\left.L_{\mathrm{p}}+\Delta l_{\mathrm{p}}-\pi\left(R_{\text {эд }}+R_{\mathrm{peд}}\right)^{2}-\right) \\
-\left(R_{\mathrm{pe}}-R_{\text {эд }}\right)^{2}
\end{array}\right)}- \\
& -\sqrt{0,25\left(L-\pi\left(R_{\text {эд }}+R_{\mathrm{pед}}\right)^{2}-\left(R_{\mathrm{peд}}-R_{\text {эд }}\right)^{2}\right)},
\end{aligned}
$$

где $R_{\text {эд }}, R_{\text {ред }}$ - радиусы шкивов электродвигателя и редуктора соответственно; $L_{\mathrm{p}}$ - длина ремня в нерастянутом состоянии; $\Delta l_{\mathrm{p}}$ - максимальное допускаемое удлинение ремня [14-16].

Совместным решением (10) и (11) относительно $\alpha_{1}$ определяется область изменения угла наклона платформы $\alpha=\left(\alpha_{0}, \alpha_{1}\left(\alpha_{0}\right)\right)$.

На рис. 5 показано, как изменяется угол наклона платформы в зависимости от положения ЭД на платформе. Конечный угол наклона платфор- мы, соответствующий максимальному допускаемому растяжению ремней, принимается равным $5^{\circ}$.

Из графика видно, что минимальный начальный угол наклона платформы к горизонтали соответствует наиболее удаленному расположению ЭД на платформе, составляя $11,5^{\circ}$. С уменьшением расстояния до ЭД вдоль платформы начальный угол наклона платформы возрастает и равен $16,2^{\circ}$ для наиболее близкого расположения ЭД вдоль платформы до оси ее вращения.

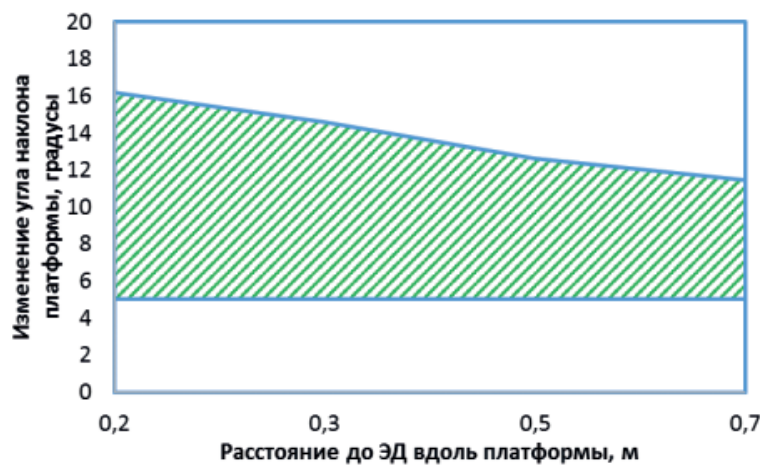

Puc.5. Изменение угла наклона платформы в процессе растяжения ремней

Fig. 5. Changing the platform inclination angle during stretching of the belts

\section{Расчет геометрических параметров} и упругих характеристик пружин

Для нахождения результирующего коэффициента упругости системы пружин запишем в дифференциальной форме закон Гука, связывающий между собой результирующую силу упругости пружин, удлинение и жесткость [17]:

$$
d F_{\text {пр }}=K_{\Sigma} d l=K_{\Sigma} L_{\mathrm{r}} \cos \alpha \cdot d \alpha,
$$

где $K_{\Sigma}$ - коэффициент упругости системы пружин; $d l$ - дифференциальное приращение длины пружин при изменении угла наклона платформы на $d \alpha$.

Изменение силы упругости пружин, обеспечивающее постоянное натяжение ремней СК при изменении угла наклона платформы, определяется нахождением дифференциала уравнения (7). Приравнивая в нем слагаемое $d F_{\text {пр }}$ к $(12)$, получаем уравнение для определения результирующей жесткости системы пружин. На рис. 6 показан графически расчет результирующего коэффициента упругости системы пружин. Из графиков на рисунке видно, что с увеличением расстояния, на котором расположен вдоль платформы ЭД, величина результирующей жесткости пружины, необходимая для поддержания постоянного натяжения ремней СК, увеличивается. Также следует отметить, что фактическое изменение результирующей силы упругости системы пружин с изменением угла наклона платформы отличается от величины, необходимой для поддержания постоянного натяжения ремней, поэтому для определения результирующей жесткости системы пружин необходимо 
рассматривать только ту область, в которой изменяется угол наклона платформы в процессе растяжения ремней (красная сплошная линия на рис. 6). Вне этой области наблюдается расхождение кривых фактического изменения результирующей силы упругости системы пружин, имеющих линейный характер (штриховые линии на рис. 6), с нелинейными кривыми изменения силы упругости, необходимой для поддержания постоянного натяжения ремней.

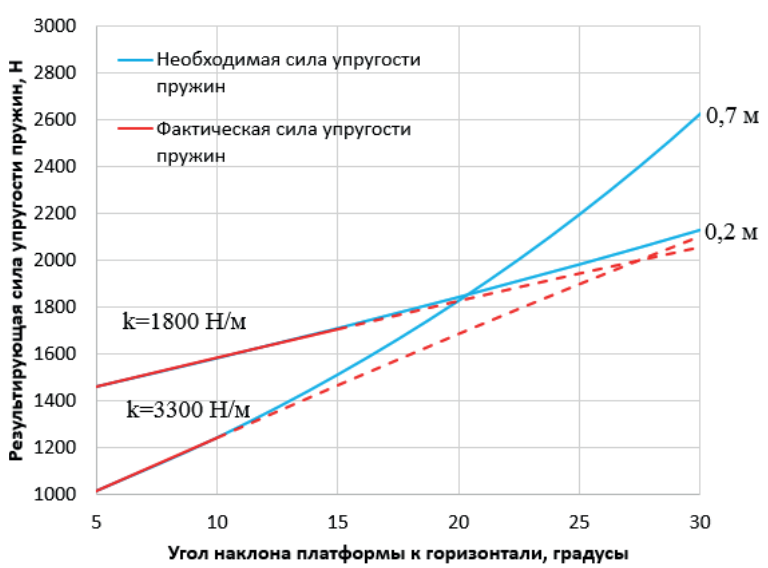

Puс.6. Расчет результирующей жесткости системы пружин при различном положении электродвигателя на плат форме

Fig. 6. Calculation of the resulting stiffness of the spring system for different positions of the motor on the platform

Для системы из двух пружин, первоначально сжатых, результирующая сила упругости запишется как

$$
F_{\text {пр }}=K_{1}\left(\Delta l_{10}-\Delta l\right)-K_{2}\left(\Delta l_{20}+\Delta l\right),
$$

где $K_{1}, \Delta l_{10}, K_{2}, \Delta l_{20}-$ коэффициенты упругости и начальные величины удлинений для верхней и нижней пружины соответственно; $\Delta l$ - приращение длины пружин в процессе растяжения ремней (при уменьшении угла наклона платформы). Из (12) и (13) следует, что результирующий коэффициент упругости системы пружин связан с коэффициентами упругости отдельной пружины соотношением

$$
K_{\Sigma}=K_{1}+K_{2}=K_{1}(1+A),
$$

где $A$ - отношение коэффициентов упругости нижней и верхней пружины соответственно.

Дальнейший расчет пружин, входящих в компоновку автоматического натяжителя, производится для случая наибольшего расстояния вдоль платформы до электродвигателя, при котором нагруженность пружин будет минимальной. На рис. 7 показано, как изменяются коэффициенты упругости пружин в зависимости от выбранной величины соотношения жесткостей $A$.

Из графиков следует, что при отсутствии нижней пружины жесткость верхней пружины равна результирующей, при этом с увеличением коэффициента упругости нижней пружины жесткость верхней уменьшается.

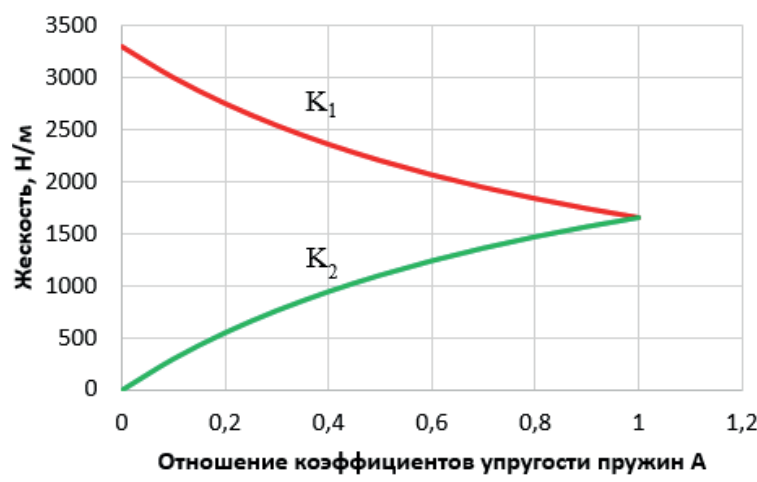

Pис. 7. Область изленения коэффициентов упругости пружин

Fig. 7. Area of change in the elasticity of springs

С учетом выбранного соотношения коэффициентов упругости пружин $A$ из (14) определяются коэффициенты упругости верхней и нижней пружин. На основании данных по величине жесткости пружин выполняется расчет их геометрических характеристик - диаметра пружины $D$, диаметра проволоки $d$, количества витков $n$. Внутренний диаметр пружины подбирается с учетом того, что внутри него должен помещаться с некоторым запасом вал упруго-деформируемой опоры. При выборе диаметра проволоки необходимо учесть, что нагруженность верхней пружины больше, чем нагруженность нижней, в результате проволока для верхней пружины должна быть более жесткой и иметь больший диаметр $[18,19]$.

Внешний диаметр пружин определяется с учетом величины внутреннего диаметра пружин согласно выражению

$$
\begin{aligned}
& D_{1}=D_{\text {в }}+\Delta D_{1}+2 d_{1} ; \\
& D_{2}=D_{\text {в }}+\Delta D_{2}+2 d_{2},
\end{aligned}
$$

где $D_{\text {в }}$ - диаметр вала упруго-деформируемой опоры; $\Delta D$ - зазор между пружиной и валом.

Необходимое количество витков для верхней и нижней пружин соответственно определяется согласно формулам [20]

$$
\begin{aligned}
& n_{1}=G_{1} \frac{d_{1}^{4}}{8 D_{1}^{3} k_{1}} ; \\
& n_{2}=G_{2} \frac{d_{2}^{4}}{8 D_{2}^{3} k_{2}},
\end{aligned}
$$

где $G$ - модуль сдвига материала пружины (зависит от марки используемой стали); $k$ - число рабочих витков в пружине.

Силы упругой деформации пружин при начальном угле наклона платформы определяются из уравнения баланса моментов сил, изменение силы упругости пружин в процессе растяжения ремней определяется изменением величины деформации пружин согласно закону Гука.

По представленной методике, базирующейся на формулах (14)-(16), выполнен расчет, результаты которого приведены в таблице. 
таблица. Параметры верхней и нижней пружины

Table. $\quad$ Parameters of the upper and lower spring

\begin{tabular}{|l|c|c|}
\hline \multicolumn{1}{|c|}{ Параметр/Parameter } & \multicolumn{2}{|c|}{ Значение/Value of } \\
\cline { 2 - 3 } & $\begin{array}{c}\text { верхней } \\
\text { пружины } \\
\text { upper spring }\end{array}$ & $\begin{array}{c}\text { нижней } \\
\text { пружины } \\
\text { lower spring }\end{array}$ \\
\hline $\begin{array}{l}\text { Диаметр проволоки, мм } \\
\text { Wire diameter, mm }\end{array}$ & 6 & 3 \\
\hline $\begin{array}{l}\text { Наружный диаметр пружины, мм } \\
\text { Spring outer diameter, mm }\end{array}$ & 70 & 65 \\
\hline $\begin{array}{l}\text { Внутренний диаметр пружины, мм } \\
\text { Spring internal diameter, mm }\end{array}$ & 58 & 59 \\
\hline Количество витков/Turning пumber & 18 & 6 \\
\hline Рабочая нагрузка, H/Work load, N & $1300-1500$ & $250-300$ \\
\hline $\begin{array}{l}\text { Коэффициент жесткости, H/м } \\
\text { Stiffnеss соеfficient, N/m }\end{array}$ & 2750 & 550 \\
\hline $\begin{array}{l}\text { Модуль сдвига материала пружины, ГПа } \\
\text { Spring таterial shear module, GPa }\end{array}$ & 78,5 & 78,5 \\
\hline
\end{tabular}

\section{Выводы}

1. Разработана методика расчета автоматического натяжителя ремней станка-качалки, включающая расчет оптимальных значений угла наклона платформы к горизонтали, положения электродвигателя на платформе и упругих характеристик пружин.

\section{СПИСОК ЛИТЕРАТУРЫ}

1. Станок-качалка с автоматическим натяжителем ремней: пат. Рос. Федерации № 163866; заявл. 05.05.2015; опубл. 10.08.2016, Бюл. № 22 .

2. Жуков К.П., Гуревич Ю.Е. Проектирование деталей и узлов машин. - М.: Изд-во «Станкин», 2004. - 671 с.

3. Приводы скважинных штанговых насосов / В.П. Жулаев, К.Р. Уразаков, М.М. Ахтямов, 3.3. Алиев. - Уфа: Изд-во УГНТУ, 2010. - $119 \mathrm{c}$

4. Balta B., Sonmez F., Cengiz A. Speed losses in V-ribbed belt drives // Mechanism and Machine Theory. -2015. - V. 86. - P. 1-14.

5. Алиев Т.М., Мелик-Шахназаров А.М., Тер-Хачатуров А.А. Измерительные информационные системы в нефтяной промышленности. - М.: Недра, 1991. - 351 с.

6. Хакимьянов М.И., Хусаинов Ф.Ф., Шафиков И.Н. Проблемы повышения энергетических характеристик электроприводов скважинных штанговых насосов // Электротехнические системы и комплексы. - 2017. - № 2 (35). - С. 35-40.

7. Takacs G., Gajda M. An Enhanced Model for the Design of Tapered Sucker-Rod Strings // Society of Petroleum Engineers. 2017. - V. 32 (Iss. 01). - P. 1-6.

8. Эксплуатация скважин установками штанговых насосов на поздней стадии разработки нефтяных месторождений / В.М. Валовский, К.М. Валовский, Г.Ю. Басос, Н.Г. Ибрагимов, В.Г. Фадеев, А.В. Артюхов. - М.: Изд-во «Нефтяное хозяйство», 2016. - $592 \mathrm{c}$.

9. Bloch H.P. Petrochemical Machinery Insights. - Woburn: Butterworth-Heinemann, 2017. -725 p.

10. Dynamic model of a Rod Pump Installation for inclined wells / R.N. Bakhtizin, K.R. Urazakov, S.F. Ismagilov, A.S. Topol'nikov, F.F. Davletshin // SOCAR Proceedings. - 2017. - № 4. P. 74-82.
2. Показано, что с точки зрения минимизации нагруженности пружин наиболее оптимальными являются: минимальное значения угла наклона платформы и максимальное расстояние вдоль нее до электродвигателя.

3. Продемонстрирован расчет коэффициентов упругости пружин, входящих в компоновку автоматического натяжителя. Результирующий коэффициент упругости системы пружин варьируется в интервале 1,8-3,3 кН/м в зависимости от положения электродвигателя вдоль платформы.

4. Рассчитаны пределы изменения угла наклона платформы в процессе растяжения приводных ремней. Начальный угол наклона платформы закономерно возрастает с уменьшением расстояния вдоль нее до электродвигателя и изменяется в пределах $11,5-16,2^{\circ}$.

5. Предлагаемый механизм автоматического натяжителя приводных ремней, расчет которого производится согласно представленной методике, позволит повысить эксплуатационную надежность станков-качалок путем обеспечения стабильной работы клиноременной передачи, способствует увеличению срока службы привода и поддержанию высокого КПД штанговой установки.

11. Mikhaylov V.G., Ponomarev A.I., Topolnikov A.S. Prediction of gas factor taking into account gas dissolved in the water at late stages development of oil fields // SOCAR Proceedings - 2017. № 3. - P. 41-48.

12. Ямалиев В.У., Ишемгужин И.Е., Латыпов Б.М. Оценка силы трения плунжера о цилиндр штангового скважинного насоса при проектировании колонны штанг // Известия Самарского научного центра Российской академии наук. - 2017. - Т. 19. № 1. - С. 70-75.

13. Ugural A.C., Fenster S.K. Advanced Strength and Applied Elasticity. - Upper Saddle River: Prentice-Hall, 2003. - 560 p.

14. Анурьев В.И. Справочник конструктора-машиностроителя (в 3-х т.). Т. 3. - М.: Изд-во «Машиностроение», 2001. - 864 с.

15. Bhandari V.B. Design of Machine Elements. - New York City: Tata McGraw-Hill Education, 2007. - $861 \mathrm{p}$.

16. Bhandari V.B. Introduction to Machine Design. - New York City: Tata McGraw-Hill Education, 2013. - 645 p.

17. Timoshenko S.P., James M.G. Theory of Elastic Stability. - Mineola: Dover Publications, 2009. - 560 p.

18. Уразаков К.P. Механизированная добыча нефти (сборник изобретений). -Уфа: Изд-во «Нефтегазовое дело», 2010. - 329 с.

19. Насосные установки для малодебитных скважин / К.Р. Уразаков, В.П. Жулаев, Ф.З. Булюкова, В.А. Молчанова. - Уфа: Изд-во УГНТУ, 2014. - 236 с.

20. Скважинные насосные установки для добычи нефти / В.Н. Ивановский, В.И. Дарищев, А.А. Сабиров, В.С. Каштанов, С.С. Пекин. - М.: ГУП Изд-во «Нефть и газ» РГУ нефти и газа им. И.М. Губкина, 2002. - 824 с.

Поступила 07.11.2018 2. 


\section{Информация об авторах}

Уразаков $\boldsymbol{K} . \boldsymbol{P}$., доктор технических наук, профессор, профессор кафедры машин и оборудования нефтегазовых промыслов Уфимского государственного нефтяного технического университета.

Тимашев Э.О., кандидат технических наук, докторант кафедры машин и оборудования нефтегазовых промыслов Уфимского государственного нефтяного технического университета.

Шайжанов Н.С., аспирант кафедры машин и оборудования нефтегазовых промыслов Уфимского государственного нефтяного технического университета.

Тугунов П.М., аспирант кафедры машин и оборудования нефтегазовых промыслов Уфимского государственного нефтяного технического университета. 
UDC 622.276.53

RESEARCH OF AN AUTOMATED SYSTEM OF TENSION OF DRIVE BELTS IN A PUMPJACK

\title{
Kamil R. Urazakov',
}

Urazakk@mail.ru

\section{Eduard 0. Timashev',}

timashev@mail.ru

\section{Nursultan S. Shayzhanov', shaizhanov007@yandex.ru}

\author{
Pavel M. Tugunov', \\ paveltugunov@gmail.com \\ 1 Ufa State Petroleum Technological University, \\ 1, Kosmonavtov street, Ufa, 450062, Russia.
}

Relevance. The efficiency of pumpjacks - the most common type of drive of sucker rod pumps - is largely determined by the reliability of the $V$-belt drive. The absence of a mechanism, which ensures optimal tension of the drive belts, causes significant energy losses in the $V$-belt transmission due to slippage of the belts and decrease in operating characteristics of pumping units. Currently, the control and adjustment of tension of the drive belts of the machine are carried out manually. The foregoing causes the relevance of developing the mechanisms for automatic belt tension.

The object: automatic tensioner of drive belts for pumpjack using the principle of adjusting the interaxle distance pulleys by changing the inclination angle of the platform with installed motor. Adjustment of the angle of inclination is achieved by the joint operation of compression springs of different rigidity. The spring of greater rigidity due to the force of the elastic compression deformation provides tension of the belts, the spring of less rigidity is designed to compensate fluctuations in the system.

The aim of the research is to study an automated system of tension of drive belts, develop a technique for calculating the automatic belt tensioner of the pumpjack, which includes determining the optimal value of the platform's inclination to the horizontal, the position of electric motor on the platform, and the elastic characteristics of the springs.

Methods: application of the principles of analytical mechanics, differential calculus for counting optimal values of automatic tension parameters.

Results. The author have developed the technique for calculating the automatic tensioner for the drive belt of a pumpjack, which makes it possible to maintain the optimum tension of the belts during operation of the sucker rod pump system. The optimum values of the initial inclination angle of the platform, the position of the electric motor on the platform, the limits of variation in the platform inclination angle during the stretching of the belts are calculated. It is shown that from the point of view of minimizing the load on the spring, the optimal values are the minimum platform inclination angle and the maximum distance along it to the electric motor. The authors calculated the compression springs included in the automatic tensioner arrangement, and determined the optimum values of the elasticity coefficients of the springs.

Key words:

Pumpjack, drive belt, belt tension, automatic tensioner, compression spring, coefficient of spring stiffness.

\section{REFERENCES}

1. Urazakov K.R., Zotov A.N., Shayzhanov N.S., Akhtyamov M.M. Stanok-kachalka s avtomaticheskim natyazhitelem remney [Pumpjack with automatic belt tensioner]. Patent RF, no. 163866,2015 .

2. Zhukov K.P., Gurevich Yu.E. Proektirovanie detaley $i$ uzlov mashin [Design of machine parts and components]. Moscow, Stankin Publ., 2004. $671 \mathrm{p}$.

3. Zhulaev V.P., Urazakov K.R., Akhtyamov M.M., Aliev Z.Z. Pri vody skvazhinnykh shtangovykh nasosov [Drives of sucker rod pumps]. Ufa, UGNT'U Publ., 2010. 119 p.

4. Balta B., Sonmez F., Cengiz A. Speed losses in V-ribbed belt drives. Mechanism and Machine Theory, 2015, vol. 86, pp. 1-14.

5. Aliev T.M., Melik-Shakhnazarov A.M., Ter-Khachaturov A.A. Izmeritelnye informatsionnye sistemy $v$ neftyanoy promyshlennosti [Measuring information systems in the oil industry]. Moscow, Nedra Publ., 1991. 351 p.

6. Khakimyanov M.I., Khusainov F.F., Shafikov I.N. Problems of increasing the energy characteristics of electric drives of borehole rod pumps. Electrotechnical systems and complexes, 2017, no. 2 (35), pp. 35-40. In Rus.
7. Takacs G., Gajda M. An Enhanced Model for the Design of Tapered Sucker-Rod Strings. Society of Petroleum Engineers, 2017, vol. 32 (Iss. 01), pp. 1-6.

8. Valovskii V.M., Valovskii K.M., Basos G.Yu., Ibragimov N.G., Fadeev V.G., Artyukhov A.V. Ekspluatatsiya skvazhin ustanovkami shtangovykh nasosou na pozdney stadii razrabotki neftyanykh mestorozhdeniy [Well operation by installation of rod pumps in the late stages of development of oil fields]. Moscow, Neftyanoe khozyaistvo Publ., 2016. 592 p.

9. Bloch H.P. Petrochemical Machinery Insights. Woburn, Butterworth-Heinemann Publ., 2017. 725 p.

10. Bakhtizin R.N., Urazakov K.R., Ismagilov S.F., Topol'nikov A.S., Davletshin F.F. Dynamic model of a Rod Pump Installation for inclined wells. SOCAR Proceedings, 2017, no 4, pp. 74-82.

11. Mikhaylov V.G., Ponomarev A.I., Topolnikov A.S. Prediction of gas factor taking into account gas dissolved in the water at late stages development of oil fields. SOCAR Proceedings, 2017, no. 3 , pp. 41-48.

12. Yamaliev V.U., Ishemzhuzhin I.E., Latypov B.M. Evaluation of the frictional force of a plunger on the cylinder of a sucker-hole 
pump in the design of a rod string. Izvestiya Samara Scientific Center, Russian Academy of Sciences, 2017, vol. 19, no. 1, pp. 70-75. In Rus.

13. Ugural A.C., Fenster S.K. Advanced Strength and Applied Elasticity. Upper Saddle River, Prentice-Hall Publ., 2003. 560 p.

14. Anuryev V.I. Spravochnik konstruktora-mashinostroitelya [Handbook of the designer-machine builder]. Moscow, Mashinostroenie Publ., 2001. Vol. 3, 864 p.

15. Bhandari V.B. Design of Machine Elements. New York, City Tata McGraw-Hill Education Publ., 2007. 861 p.

16. Bhandari V.B. Introduction to Machine Design. New York City, Tata McGraw-Hill Education Publ., 2013. 645 p.

17. Timoshenko S.P., James M.G. Theory of Elastic Stability. Mineola, Dover Publications Publ., 2009. 560 p.
18. Urazakov K.R. Mekhanizirovannaya dobycha nefti (sbornik izobretenii) [Mechanized oil production (collection of Inventions)]. Ufa, Neftegazovoe delo Publ., 2010. 329 p.

19. Urazakov K.R., Zhulaev V.P., Bulyukova F.Z., Molchanova V.A. Nasosnye ustanovki dlya malodebitnykh skvazhin [Pumping installations for low-yield wells]. Ufa, UGNTU Publ., 2014. 236 p.

20. Ivanovskii V.N., Darishchev V.I., Sabirov A.A., Kashtanov V.S., Pekin S.S. Skvazhinnye nasosnye ustanovki dlya dobychi nefti [Downhole pumping units for oil production]. Moscow, GUP «Neft i gaz» RGU nefti i gaza im. I.M. Gubkina Publ., 2002. $824 \mathrm{p}$.

\section{Information about the authors}

Kamil R. Urazakov, Dr. Sc., professor, Ufa State Petroleum Technological University.

Eduard 0. Timashev, Cand. Sc., doctoral candidate, Ufa State Petroleum Technological University.

Nursultan S. Shayzhanov, post-graduate student, Ufa State Petroleum Technological University.

Pavel M. Tugunov, post-graduate student, Ufa State Petroleum Technological University. 